\title{
Neutral stochastic delay partial functional integro-differential equations driven by a fractional Brownian motion
}

\author{
Tomás Caraballo* \\ Dpto. Ecuaciones Diferenciales y Análisis Numérico, Universidad de Sevilla \\ Apdo. de Correos 1160, 41080-Sevilla, Spain \\ Mamadou Abdoul Diop ${ }^{\dagger}$ \\ Université Gaston Berger de Saint-Louis, UFR SAT, Département de Mathématiques, \\ 234, Saint-Louis, Sénégal
}

\begin{abstract}
This paper deals with the existence and uniqueness of mild solutions to neutral stochastic delay functional integro-differential equations perturbed by a fractional Brownian motion $B^{H}$, with Hurst parameter $H \in\left(\frac{1}{2}, 1\right)$. We use the theory of resolvent operators developed in R. Grimmer [5] to show the existence of mild solutions. An example is provided to illustrate the results of this work.
\end{abstract}

2010 MSC: 60H15; 35R60.

Keys words: Resolvent operators, $C_{0}$-semigroup, Wiener process, mild solutions, Fractional Brownian motion.

\section{Introduction}

In this paper we are interested in the existence of solutions for the following stochastic neutral partial functional integro-differential equations with finite delay

$$
\left\{\begin{aligned}
d[u(t) & +G(t, u(t-r(t)))]=A[u(t)+G(t, u(t-r(t)))] d t \\
& +\left[\int_{0}^{t} B(t-s)[u(s)+G(s, u(s-r(s)))] d s+F(t, u(t-\delta(t)))\right] d t \\
& +\sigma(t) d B^{H}(t) \quad \text { for } t \in[0, T] \\
u_{0}(.) & =\varphi, \quad-r \leq t \leq 0
\end{aligned}\right.
$$

where $A$ is the infinitesimal generator of a strongly continuous semigroup $(T(t))_{t \geq 0}$ on a Hilbert space $X$ with domain $D(A)$. Here $B(t)$ is a closed linear operator on $X$ with domain $D(B) \supset$

*E-mail address: caraball@us.es (Corresponding author)

${ }^{\dagger}$ E-mail address: mamadou-abdoul.diop@ugb.edu.sn 
$D(A)$ which is independent of $t ; B^{H}$ is a fractional Brownian motion on a real and separable Hilbert space $Y, r, \delta:[0,+\infty[\rightarrow[0, \tau](\tau>0)$ are continuous functions, and $G, F:[0,+\infty[\times X \rightarrow$ $X, \sigma:\left[0,+\infty\left[\times \mathcal{L}_{2}^{0}(Y, X)\right.\right.$ are appropriate functions. Here $\mathcal{L}_{2}^{0}(Y, X)$ denotes the space of all $Q$-Hilbert-Schmidt operators from $Y$ into $X$ (see Section 2).

Neutral differential equations have many applications in physical and biological systems, for this reason these equations have received much attention in recent years (see, e.g. [8],[2], [7],[13], [15],[16] and references therein). The literature related to neutral differential equations of the type (1.1) is not vast. Recently in [1], it was established the existence and the asymptotic behaviour of solutions of the following neutral stochastic differential equation with finite delay

$$
\left\{\begin{array}{l}
d[u(t)+G(t, u(t-r(t)))]=[A u(t)+F(t, u(t-\delta(t)))] d t+\sigma(t) d B^{H}(t) \quad \text { for } t \in[0, T], \\
u_{0}(t)=\varphi(t), \quad-\tau \leq t \leq 0
\end{array}\right.
$$

where $A$ generates an analytic semigroup of bounded linear operators $(S(t))_{t \geq 0}$ in a Hilbert space $X$, and $G, F:[0,+\infty[\times X \rightarrow X$.

The goal of this work is to extend this problem to neutral integro-differential type equation and to discuss the existence of solutions for Eq. (1.1) by using the resolvent operators. There is a rich theory for analytic semigroups and we wish to develop theories for (1.1) which yield analytic resolvent. However, our objective in the present paper is to apply the theory developed by Grimmer in [5], because it is valid for generators of strongly continuous semigroups, not necessarily analytic. This fact motivates that the equation under study must have the special form (1.1). A more natural extension of (1.1), namely

$$
\left\{\begin{array}{c}
d[u(t)+G(t, u(t-r(t)))]=\left[A u(t)+\int_{0}^{t} B(t-s) u(s) d s+F(t, u(t-\delta(t)))\right] d t \\
\quad+\sigma(t) d B^{H}(t) \quad \text { for } t \in[0, T], \\
u_{0}(t)=\varphi(t), \quad-\tau \leq t \leq 0,
\end{array}\right.
$$

will be analyzed in a subsequent paper under stronger assumptions on the operator $A$.

Let us describe the content of the paper. In Section 2, we introduce some notations, concepts of resolvent operators, basic results about fractional Brownian motion, Wiener integral over Hilbert space. In Section 3, we study the existence and uniqueness of mild solutions for the system (1.1) with their proofs. In section 4 , we provide an example to illustrate our previous abstract results.

\section{Wiener Process and deterministic integrodifferential equations}

\subsection{Wiener process}

In this section we introduce the fractional Brownian motion as well as the Wiener integral with respect to it. We also establish some important results which will be needed throughout the paper. Let $(\Omega, \mathcal{F}, \mathbb{P})$ be a complete probability space.

Definition 2.1. Given $H \in(0,1)$, a continuous centered Gaussian process $\beta^{H}(t), t \in \mathbb{R}$, with covariance function

$$
R_{H}(s, t)=\mathbb{E}\left[\beta^{H}(t) \beta^{H}(s)\right]=\frac{1}{2}\left(t^{2 H}+s^{2 H}-|t-s|^{2 H}\right), \quad t, s \in \mathbb{R}
$$

is called a two-sided one-dimensional fractional Brownian motion ( $\mathrm{fBm}$ ), and $H$ is the Hurst parameter. 
Now we aim at introducing the Wiener integral with respect to the one-dimensional $\mathrm{fBm} \beta^{H}$. Let $T>0$ and denote by $\Lambda$ the linear space of $\mathbb{R}$-valued step function on $[0, T]$, that is $\phi \in \Lambda$ if

$$
\phi(t)=\sum_{i=1}^{n-1} x_{i} 1_{\left[t_{i}, t_{i+1}\right)}(t)
$$

where $t \in[0, T], x_{i} \in \mathbb{R}$ and $0=t_{1}<t_{2}<\cdots<t_{n}=T$. For $\phi \in \Lambda$ we define its Wiener integral with respect to $\beta^{H}$ as

$$
\int_{0}^{T} \phi(s) d \beta^{H}(s)=\sum_{i=1}^{n-1} x_{i}\left(\beta^{H}\left(t_{i+1}\right)-\beta^{H}\left(t_{i}\right)\right) .
$$

Let $\mathcal{H}$ be the Hilbert space defined as the closure of $\Lambda$ with respect to the scalar product $\left\langle 1_{[0 ; t]}, 1_{[0 ; s]}\right\rangle_{\mathcal{H}}=R_{H}(t, s)$.

Then the mapping

$$
\phi=\sum_{i=1}^{n-1} x_{i} 1_{\left[t_{i}, t_{i+1}\right)} \rightarrow \int_{0}^{T} \phi(s) d \beta^{H}(s)
$$

is an isometry between $\Lambda$ and the linear space $\operatorname{span}\left\{\beta^{H}, t \in[0, T]\right\}$, which can be extended to an isometry between $\mathcal{H}$ and the first Wiener chaos of the $\mathrm{fBm} \overline{\operatorname{span}}^{2}(\Omega)\left\{\beta^{H}, \quad t \in[0, T]\right\}$ (see [3]). The image of an element $\varphi \in \mathcal{H}$ by this isometry is called the Wiener integral of $\varphi$ with respect to $\beta^{H}$. Our next goal is to give an explicit expression of this integral. To this end, consider the Kernel

$$
K_{H}(t, s)=c_{H} s^{\frac{1}{2}-H} \int_{s}^{t}(u-s)^{H-\frac{3}{2}} u^{H-\frac{1}{2}} d u
$$

where $c_{H}=\sqrt{\frac{H(2 H-1)}{B\left(2-2 H, H-\frac{1}{2}\right)}}$, with $B$ denoting the Beta function and $t \leq s$. It is not difficult to see that

$$
\frac{\partial K_{H}}{\partial t}(t, s)=c_{H}\left(\frac{t}{s}\right)^{\frac{1}{2}-H}(t-s)^{H-\frac{3}{2}} .
$$

Consider the linear operator $K_{H}^{*}: \Lambda \longrightarrow L^{2}([0, T])$ given by

$$
\left(K_{H}^{*} \varphi\right)(s)=\int_{s}^{t} \varphi(t) \frac{\partial K}{\partial t}(t, s) d t .
$$

Then

$$
K_{H}^{*} 1_{[0 ; t]}(s)=K_{H}(t, s) 1_{[0 ; t]}(s)
$$

and $K_{H}^{*}$ is an isometry between $\Lambda$ and $L^{2}([0, T])$ that can be extended to $\Lambda$ (see [11]).

Considering $W=\{W(t), \quad t \in[0, T]\}$ defined by

$$
W(t)=\beta^{H}\left(\left(K_{H}^{*}\right)^{-1} 1_{[0 ; t]}\right),
$$

it turns out that $W$ is a Wiener process and $\beta^{H}$ has the following Wiener integral representation:

$$
\beta^{H}(t)=\int_{0}^{t} K_{H}(t, s) d W(s) .
$$

In addition, for any $\varphi \in \Lambda$,

$$
\int_{0}^{T} \varphi(s) d \beta^{H}(s)=\int_{0}^{T}\left(K_{H}^{*} \varphi\right)(t) d W(t)
$$


if and only if $K_{H}^{*} \varphi \in L^{2}([0, T])$.

Also denoting $L_{\mathcal{H}}^{2}([0, T])=\left\{\varphi \in \Lambda, K_{H}^{*} \varphi \in L^{2}([0, T])\right\}$, since $H>\frac{1}{2}$, we have

$$
L^{\frac{1}{H}}([0, T]) \subset L_{\mathcal{H}}^{2}([0, T]),
$$

see [12]. Moreover, the following useful result holds:

Lemma 2.2. (Nualart [14]) For $\varphi \in L^{\frac{1}{H}}([0, T])$,

$$
\left.H(2 H-1) \int_{0}^{T} \int_{0}^{T} \mid \varphi(r) \| \varphi(u)\right)\left\|r-\left.u\right|^{2 H-2} d r d u \leq c_{H}\right\| \varphi \|_{L^{\frac{1}{H}([0, T]}}^{2} .
$$

Next we are interested in considering a $\mathrm{fBm}$ with values in a Hilbert space and giving the definition of the corresponding stochastic integral.

Let $\left(X,\|\cdot\|_{X},(., .)_{X}\right)$ and $\left(Y,\|\cdot\|_{Y},(., .)_{Y}\right)$ be separable Hilbert spaces. Let $\mathcal{L}(Y, X)$ denote the space of all bounded linear operator from $Y$ to $X$. Let $Q \in \mathcal{L}(Y, X)$ be a non-negative self-adjoint operator. Denote by $L_{Q}^{0}(Y, X)$ the space of $\vartheta \in L(Y, X)$ such that $\vartheta Q^{\frac{1}{2}}$ is a Hilbert-Schmidt operator. The norm is given by

$$
|\vartheta|_{L_{Q}^{0}(Y, X)}^{2}=\left|\vartheta Q^{\frac{1}{2}}\right|_{H S}=\operatorname{tr}\left(\vartheta Q \vartheta^{*}\right) .
$$

Then $\vartheta$ is called a $Q$-Hilbert-Schmidt operator from $Y$ to $X$.

Let $\left\{\beta_{n}^{H}(t)\right\}_{n \in \mathbb{N}}$ be a sequence of two-sided one-dimensional standard fractional Brownian motions mutually independent on $(\Omega, \mathcal{F}, \mathbb{P})$. When one considers the following series

$$
\sum_{n=1}^{\infty} \beta_{n}^{H}(t) e_{n}, \quad t \geq 0
$$

where $\left\{e_{n}\right\}_{n \in \mathbb{N}}$ is a complete orthonormal bais in $X$, this series does not necessarily converge in the space $Y$. Thus we consider a $Y$-valued stochastic process

$$
B_{Q}^{H}(t)=\sum_{n=1}^{\infty} \beta_{n}^{H}(t) Q^{\frac{1}{2}} e_{n}, \quad t \geq 0 .
$$

If $Q$ is a non-negative self-adjoint trace class operator, this series converges in the space $Y$, that is, it holds that $B_{Q}^{H}(t) \in L^{2}(\Omega, Y)$. Then, we say that the above $B_{Q}^{H}(t)$ is a $Y$-valued $Q$-cylindrical fractional Brownian motion with covariance operator $Q$. For example, if $\left\{\sigma_{n}\right\}_{n \in \mathbb{N}}$ is a bounded sequence of non-negative real numbers such that $Q e_{n}=\sigma_{n} e_{n}$, assuming that $Q$ is a nuclear operator in $Y$ (that is, $\left.\sum_{n=1}^{\infty} \sigma_{n}<\infty\right)$, then the stochastic process

$$
B_{Q}^{H}(t)=\sum_{n=1}^{\infty} \beta_{n}^{H}(t) Q^{\frac{1}{2}} e_{n}=\sum_{n=1}^{\infty} \sqrt{\sigma_{n}} \beta_{n}^{H}(t) e_{n}, \quad t \geq 0,
$$

is well-defined as a $Y$-valued $Q$-cylindrical fractional Brownian motion. Let $\varphi:[0, T] \rightarrow L_{Q}^{0}(Y, X)$ such that

$$
\sum_{n=1}^{\infty}\left\|K_{H}^{*}\left(\varphi Q^{\frac{1}{2}} e_{n}\right)\right\|_{\mathcal{L}^{2}([0, T] ; X)}<\infty
$$


Definition 2.3. Let $\varphi:[0, T] \rightarrow L_{H}^{0}(Y, X)$ satisfy $(2.2)$. Then, its stochastic integral with respect to the $\mathrm{fBm} B_{Q}^{H}$ is defined, for $t \geq 0$, as follows

$$
\int_{0}^{t} \varphi(s) d B_{Q}^{H}(s):=\sum_{n=1}^{\infty} \int_{0}^{t} \varphi(s) Q^{\frac{1}{2}} e_{n} \beta_{n}^{H}(s)=\sum_{n=1}^{\infty} \int_{0}^{t}\left(K_{H}^{*}\left(\varphi Q^{\frac{1}{2}} e_{n}\right)\right)(s) d W(s) .
$$

Notice that if

$$
\sum_{n=1}^{\infty}\left\|\varphi Q^{\frac{1}{2}} e_{n}\right\|_{L \frac{1}{H}([0, T] ; X)}<\infty
$$

then in particular (2.2) holds, which follows immediately from (2.1).

Now we end this subsection by stating the following result which is fundamental to prove our result. It can proved by similar arguments as those used to prove Lemma 2 in Caraballo et al.[10].

Lemma 2.4. If $\psi:[0, T] \rightarrow \mathcal{L}_{2}^{0}(Y, X)$ satisfies $\int_{0}^{T}\|\psi\|_{\mathcal{L}_{2}^{0}}^{2} d s<\infty$ then the above sum in (2.3) is well defined as a $X$-valued random variable and we have

$$
E\left\|\int_{0}^{t} \psi(s) d B^{H}(s)\right\|^{2} \leq 2 H t^{2 H-1} \int_{0}^{t}\|\psi(s)\|_{\mathcal{L}_{2}^{0}}^{2} d s
$$

Proof. See [1].

\subsection{Partial integro-differential equations in Banach spaces}

In this section, we recall some fundamental results needed to establish our results. Regarding the theory of resolvent operators we refer the reader to [5]. Throughout the paper, $X$ is a Banach space, $A$ and $B(t)$ are closed linear operators on $X$. $Y$ represents the Banach space $D(A)$ equipped with the graph norm defined by

$$
|y|_{Y}:=|A y|+|y| \quad \text { for } y \in Y .
$$

The notations $C([0,+\infty) ; Y), \mathcal{B}(Y, X)$ stand for the space of all continuous functions from $[0,+\infty)$ into $Y$, the set of all bounded linear operators from $Y$ into $X$, respectively. We consider the following Cauchy problem

$$
\left\{\begin{array}{l}
v^{\prime}(t)=A v(t)+\int_{0}^{t} B(t-s) v(s) d s, \quad \text { for } t \geq 0 \\
v(0)=v_{0} \in X
\end{array}\right.
$$

Definition 2.5. ([5]). A resolvent operator for Eq. (2.4) is a bounded linear operator valued function $R(t) \in \mathcal{L}(X)$ for $t \geq 0$, satisfying the following properties:

(i) $R(0)=I$ and $|R(t)| \leq N e^{\beta t}$ for some constants $\mathrm{N}$ and $\beta$.

(ii) For each $x \in X, R(t) x$ is strongly continuous for $t \geq 0$.

(iii) $R(t) \in \mathcal{L}(Y)$ for $t \geq 0$. For $x \in Y, R(\cdot) x \in C^{1}([0,+\infty) ; X) \cap C([0,+\infty) ; Y)$ and

$$
\begin{aligned}
R^{\prime}(t) x & =A R(t) x+\int_{0}^{t} B(t-s) R(s) x d s \\
& =R(t) A x+\int_{0}^{t} R(t-s) B(s) x d s \quad \text { for } t \geq 0
\end{aligned}
$$


The resolvent operators play an important role to study the existence of solutions and to give a variation of constants formula for nonlinear systems. We need to know when the linear system (2.4) has a resolvent operator. For more details on resolvent operators, we refer the reader to [5]. The following theorem gives a satisfactory answer to this problem and it will be used in this work to develop our main results.

In what follows we suppose the following assumptions :

(H1) A is the infinitesimal generator of a strongly continuous semigroup $\{T(t)\}_{t \geq 0}$ on $\mathrm{X}$.

(H2) For all $t \geq 0, B(t)$ is a closed linear operator from $D(A)$ to $\mathrm{X}$, and $B(t) \in \mathcal{B}(Y, X)$. For any $y \in Y$, the map $t \rightarrow B(t) y$ is bounded, differentiable and the derivative $t \rightarrow B^{\prime}(t) y$ is bounded and uniformly continuous on $\mathbb{R}^{+}$.

Theorem 2.6. ([5, Theorem 3.7]) Assume that (H1)-(H2) hold. Then there exists a unique resolvent operator for the Cauchy problem (2.4).

In what follows, we establish some results for the existence of solutions of the following integro-differential equation

$$
\left\{\begin{array}{l}
v^{\prime}(t)=A v(t)+\int_{0}^{t} B(t-s) v(s) d s+q(t), \text { for } t \geq 0 \\
v(0)=v_{0} \in X
\end{array}\right.
$$

where $q:[0,+\infty[\rightarrow X$ is a continuous function.

Definition 2.7. ([5]). A continuous function $v:[0,+\infty) \rightarrow X$ is said to be a strict solution of Eq. (2.5) if

(i) $v \in C^{1}([0,+\infty) ; X) \cap C([0,+\infty) ; Y)$,

(ii) $v$ satisfies Eq. (2.5) for $t \geq 0$.

Remark 2.8. From this definition, we deduce that $v(t) \in D(A)$, and the function $B(t-s) v(s)$ is integrable, for all $t \geq 0$ and $s \in[0, t]$.

Theorem 2.9. ([5, Theorem 2.5]). Assume that (H1)-(H2) hold. If $v$ is a strict solution of Eq. (2.5), then

$$
v(t)=R(t) v_{0}+\int_{0}^{t} R(t-s) q(s) d s \quad \text { for } \quad t \geq 0 .
$$

Accordingly, we have the following definition.

Definition 2.10. ([5]). A function $v:[0,+\infty) \rightarrow X$ is called a mild solution of (2.5) if $v$ satisfies the variation of constants formula $(2.6)$, for $v_{0} \in X$.

The next theorem provides sufficient conditions for the regularity of solutions of Eq. (2.5). Namely we establish a sufficient condition ensuring when a mild solution is a strict one.

Theorem 2.11. ([5, Corollary 3.8]) Let $q \in C^{1}([0,+\infty) ; X)$ and $v$ be defined by (2.6). If $v_{0} \in D(A)$, then $v$ is a strict solution of Eq. (2.5). 


\section{Existence of mild solutions for Eq (1.1)}

In this section, we establish the existence and uniqueness of mild solutions of Eq. (1.1) under Lipschitz conditions. We use the following hypotheses to prove our results.

(H3) The function $F:[0,+\infty[\times X \rightarrow X$ satisfies the following conditions: there exist positive constant $C_{1}, C_{2}$ such that, for all $t \in[0, T]$ and $x, y \in X$

$$
\begin{gathered}
\|F(t, x)-F(t, y)\| \leq C_{1}\|x-y\|, \\
\|F(t, x)\|^{2} \leq C_{2}\left(1+\|x\|^{2}\right) .
\end{gathered}
$$

(H4) The function $G:[0,+\infty[\times X \rightarrow X$ satisfies the following conditions: there exist positive constant $C_{3}, C_{4}, 0<C_{3}<1$ such that, for all $t \in[0, T]$ and $x, y \in X$

$$
\begin{gathered}
\|G(t, x)-G(t, y)\| \leq C_{3}\|x-y\|, \\
\|G(t, x)\|^{2} \leq C_{4}\left(1+\|x\|^{2}\right) .
\end{gathered}
$$

(H5) The function $G$ is continuous in the quadratic mean sense:

For all $x \in u \in \mathcal{C}\left([0, T], L^{2}(\Omega, X)\right), \quad \lim _{t \rightarrow s} \mathbb{E}\|G(t, x(t))-G(s, x(s))\|^{2}=0$.

(H6) The function $\sigma:\left[0,+\infty\left[\rightarrow \mathcal{L}_{2}^{0}(Y, X)\right.\right.$ satisfies

$$
\int_{0}^{T}\|\sigma(s)\|_{\mathcal{L}_{2}^{0}}^{2} d s<\infty, \quad \forall T>0 .
$$

Moreover, we assume that $\varphi \in \mathcal{C}\left([-\tau, 0], L^{2}(\Omega, X)\right)$.

We now introduce the concept of mild solution of the Eq.(1.1).

Definition 3.1. An $X$-valued process $\{u(t), t \in[-\tau, T]\}$, is called a mild solution of Eq.(1.1) if $u \in \mathcal{C}\left([-\tau, 0], L^{2}(\Omega, X)\right), u(t)=\varphi(t)$ for $t \in[-\tau, 0]$, and, for $t \in[0, T]$, satisfies

$$
\begin{aligned}
u(t)+G(t, u(t-r(t)))= & R(t)[\varphi(0)-G(0, \varphi(-r(0)))]+\int_{0}^{t} R(t-s) F(s, u(s-\delta(s))) d s \\
& +\int_{0}^{t} R(t-s) \sigma(s) d B^{H}(s) \mathbb{P}-\text { a.s. }
\end{aligned}
$$

To show our main results we first recall the following Lemma.

Lemma 3.2. (Caraballo et al. [9, Lemma 1]). For $x, y \in X$ and $0<c<1$,

$$
\|x\|_{X}^{2} \leq \frac{1}{1-c}\|x-y\|_{X}^{2}+\frac{1}{c}\|y\|_{X}^{2} .
$$

Theorem 3.3. Under the assumptions (H1)-(H6), for every $\varphi \in \mathcal{C}\left([-\tau, T], L^{2}(\Omega, X)\right)$ there exists a unique mild solution $u$ to Eq. (1.1).

Proof. Assume that $T>0$ is a fixed time and let $C_{T}:=\mathcal{C}\left([-\tau, T], L^{2}(\Omega, X)\right)$ be the Banach space of all continuous functions from $[-\tau, T]$ into $L^{2}(\Omega, X)$ equipped with the supremum norm $\|\zeta\|_{C_{T}}=\sup _{z \in[-\tau, T]}\left(\mathbb{E}\|\zeta(z)\|^{2}\right)^{1 / 2}$ and let us consider the set

$$
S_{T}(\varphi):=\left\{u \in \mathcal{C}\left([-\tau, 0], L^{2}(\Omega, X)\right): u(s)=\varphi(s), \text { for } s \in[-\tau, 0]\right\} .
$$


$S_{T}(\varphi)$ is a closed subset of $C_{T}$ provided with the norm $\|\cdot\|_{C_{T}}$. Define the operator $\Gamma$ on $S_{T}(\varphi)$ by $\Gamma(u)(t)=\varphi(t)$ for $t \in[-\tau, 0]$, and for $t \in[0, T]$

$$
\begin{aligned}
\Gamma(u)(t)= & R(t)[\varphi(0)-G(0, \varphi(-r(0)))]-G(t, u(t-r(t))) \\
& +\int_{0}^{t} R(t-s) F(s, u(s-\delta(s))) d s+\int_{0}^{t} R(t-s) \sigma(s) d B^{H}(s) .
\end{aligned}
$$

Then it is clear that, proving the existence of mild solutions to Eq. (1.1) is equivalent to finding a fixed point for the operator $\Gamma$.

Next we will show, by using the Banach fixed point theorem that $\Gamma$ has a fixed point. We split the proof into two steps.

Step 1: For arbitrary $u \in S_{T}(\varphi)$, let us prove that $t \rightarrow \Gamma(u)(t)$ is continuous on the intervall $[0, T]$ in the $L^{2}(\Omega, X)$-sense. Let $0<t<T$ and $|h|$ be sufficiently small. Then, for any fixed $u \in S_{T}(\varphi)$, we have

$$
\begin{aligned}
& \|(\Gamma(u)(t+h)-(\Gamma(u)(t) \| \\
& \leq\|(R(t+h)-R(t))[\varphi(0)-G(0, \varphi(-r(0)))]\| \\
& \quad+\|G(t+h, u(t+h-r(t+h)))-G(t, u(t-r(t)))\| \\
& \quad+\left\|\int_{0}^{t+h} R(t+h-s) F(s, u(s-\delta(s))) d s-\int_{0}^{t} R(t-s) F(s, u(s-\tau(s))) d s\right\| \\
& \quad+\left\|\int_{0}^{t+h} R(t+h-s) \sigma(s) d B^{H}(s)-\int_{0}^{t} R(t-s) \sigma(s) d B^{H}(s)\right\| \\
& =\sum_{1 \leq i \leq 4} I_{i}(h) .
\end{aligned}
$$

Using the property (ii) of Definition 2.5, we obtain

$$
\lim _{h \rightarrow 0}(R(t+h)-R(t))(\varphi(0)-G(0, \varphi(-r(0))))=0 .
$$

Without loss of generality, we can assume that $\beta>0$. Using the property (i) of Definition 2.5 , we obtain

$$
\|(R(t+h)-R(t))(\varphi(0)-G(0, \varphi(-r(0))))\| \leq\left[N e^{\beta(t+h)}+N e^{\beta t}\right]\|\varphi(0)-G(0, \varphi(-r(0)))\| .
$$

Then, by the Lebesgue majorant Theorem, we conclude that

$$
\lim _{h \rightarrow 0} \mathbb{E}\left|I_{1}(h)\right|^{2}=0 .
$$

Moreover, assumption (H5) ensures that

$$
\lim _{h \rightarrow 0} \mathbb{E}\left|I_{2}(h)\right|^{2}=0 .
$$

For the third term $I_{3}(h)$, we suppose $h>0$ (similar estimates hold for $h<0$ ), then we have

$$
\begin{aligned}
I_{3}(h) & \leq\left\|\int_{0}^{t}(R(t+h-s)-R(t-s)) F(s, u(s-\delta(s))) d s\right\|+\left\|\int_{t}^{t+h} R(t+h-s) F(s, u(s-\delta(s))) d s\right\| \\
& \leq I_{31}(h)+I_{32}(h)
\end{aligned}
$$


Thanks to Hölder's inequality,

$$
\mathbb{E}\left|I_{31}(h)\right|^{2} \leq t \mathbb{E} \int_{0}^{t}\|(R(t+h-s)-R(t-s)) F(s, u(s-\delta(s)))\|^{2} d s .
$$

Again exploiting properties (i) and (ii) of Definition 2.5, we have for each $s \in[0, t]$,

$$
\lim _{h \rightarrow 0}(R(t+h-s)-R(t-s)) F(s, u(s-\delta(s)))=0,
$$

and

$$
\|(R(t+h-s)-R(t-s)) F(s, u(s-\delta(s))) d s\| \leq \tilde{N}\|F(s, u(s-\delta(s))) d s\| \in \mathbb{L}^{2}([0, t] \times \Omega),
$$

where $\tilde{N}=\left[2 N^{2} e^{2 \beta(t+h)}+2 N^{2} e^{2 \beta t}\right]$. Then, by the Lebesgue Majorant Theorem, we conclude that

$$
\lim _{h \rightarrow 0} \mathbb{E}\left|I_{31}(h)\right|^{2}=0 .
$$

Next, using property (ii) of Definition 2.5 and Hölder's inequality, it follows

$$
\mathbb{E}\left|I_{32}(h)\right|^{2} \leq C_{2}^{2} h N^{2} e^{\beta T} \int_{0}^{T}\left(\mathbb{E}\|u(s-\delta(s))\|^{2}+1\right) d s,
$$

and then

$$
\lim _{h \rightarrow 0} \mathbb{E}\left|I_{32}(h)\right|^{2}=0
$$

Now, for the term $I_{4}(h)$, we have

$$
\begin{aligned}
I_{4}(h) & \leq\left\|\int_{0}^{t}(R(t+h-s)-R(t-s)) \sigma(s) d B^{H}(s)\right\|+\left\|\int_{t}^{t+h} R(t+h-s) \sigma(s) d B^{H}(s)\right\| \\
& \leq I_{41}(h)+I_{42}(h) .
\end{aligned}
$$

By Lemma 2.4,

$$
\mathbb{E}\left|I_{41}(h)\right|^{2} \leq 2 H t^{2 H-1} \int_{0}^{t}\|(R(t+h-s)-R(t-s)) \sigma(s)\|_{\mathcal{L}_{2}^{0}}^{2}
$$

Since $\lim _{h \rightarrow 0}\|(R(t+h-s)-R(t-s)) \sigma(s)\|_{\mathcal{L}_{2}^{0}}^{2}=0$ and

$$
\|(R(t+h-s)-R(t-s)) \sigma(s)\|_{\mathcal{L}_{2}^{0}} \leq\left[N^{2} e^{2 \beta(t+h)}+N^{2} e^{2 \beta t}\right]\|\sigma(s)\|_{\mathcal{L}_{2}^{0}}^{2} \in \mathbb{L}^{1}([0, T], d s),
$$

the Lebesgue majorant Theorem implies

$$
\lim _{h \rightarrow 0} \mathbb{E}\left|I_{41}(h)\right|^{2}=0 .
$$

Again by Lemma 2.4, we obtain that

$$
\mathbb{E}\left|I_{42}(h)\right|^{2} \leq 2 H N^{2} e^{\beta(t+h)} h^{2 H-1} \int_{t}^{t+h}\|\sigma(s)\|_{\mathcal{L}_{2}^{0}} d s \rightarrow 0 \quad \text { when } h \rightarrow 0 .
$$

The above arguments show that $\left.\lim _{h \rightarrow 0} \mathbb{E} \| \Gamma(u)(t+h)-\Gamma(u)(t)\right) \|^{2}=0$. Hence, we conclude that the function $t \rightarrow \Gamma(u)(t)$ is continuous on $[0, T]$ in the $L^{2}$-sense.

Step 2: Now we show that $\Gamma$ is a contracting mapping in $S_{T_{1}}(\varphi)$ for some small enough $T_{1}<T$. 
For every $u, v \in S_{T}(\varphi)$ and $t \in[0, T]$, by using Lemma 3.2 we obtain

$$
\begin{aligned}
\|\Gamma(u)(t)-\Gamma(v)(t)\|^{2} \leq & \frac{1}{C_{3}}\|G(t, u(t-r(t)))-G(t, v(t-r(t)))\|^{2} \\
& +\frac{1}{1-C_{3}}\left\|\int_{0}^{t} R(t-s)(F(s, u(s-\delta(s)))-F(s, v(s-\delta(s)))) d s\right\|^{2}
\end{aligned}
$$

Owing to the Lipschitz properties of $F$ and $G$ combined with Hölder's inequality, we obtain

$$
\begin{aligned}
\mathbb{E}\|\Gamma(u)(t)-\Gamma(v)(t)\|^{2} \leq & C_{3} \mathbb{E}\|u(t-r(t))-v(t-r(t))\|^{2} \\
& +\frac{1}{1-C_{3}} N^{2} C_{1}^{2} e^{2 \beta t} \beta^{-1} t \int_{0}^{t} \mathbb{E}\|u(s-\delta(s))-v(s-\delta(s))\|^{2} d s
\end{aligned}
$$

Hence

$$
\sup _{s \in[-\tau, t]} \mathbb{E}\|\Gamma(u)(t)-\Gamma(v)(t)\|^{2} \leq \alpha(t) \sup _{s \in[-\tau, t]} \mathbb{E}\|u(s)-v(s)\|^{2}
$$

where $\alpha(t)=C_{3}+\frac{1}{1-C_{3}} N^{2} C_{1}^{2} e^{2 \beta t} \beta^{-1} t$.

By condition (iii) in (H4) we have $\alpha(0)=C_{3}<1$. Then there exists $0<T_{1} \leq T$ such that $0<\alpha\left(T_{1}\right)<1$ and $\Gamma$ is a contraction mapping on $S_{T_{1}}(\varphi)$ and therefore has a unique fixed point, which is a mild solution of Eq. (1.1) on $\left[-\tau, T_{1}\right]$. This procedure can be repeated a finite number of times in order to extend the solution to the entire interval $[-\tau, T]$. This completes the proof.

\section{Application}

Neutral stochastic differential equations arise in many real world problems such as physics, population dynamics, ecology, biological systems, biotechnology, optimal control, theory of elasticity, electrical networks, and so forth. We consider the following stochastic partial neutral functional integro-differential equation with finite delays $r_{1}, r_{2}\left(\infty>\tau>r_{i} \geq 0, i=1,2\right)$ :

$$
\left\{\begin{aligned}
& \frac{\partial}{\partial t}[x(t, \xi)+\left.g\left(t, x\left(t-r_{1}, \xi\right)\right)\right]=\frac{\partial^{2}}{\partial \xi^{2}}\left[x(t, \xi)+g\left(t, x\left(t-r_{1}, \xi\right)\right)\right] \\
&+\int_{0}^{t} b(t-s) \frac{\partial^{2}}{\partial \xi^{2}}\left[x(s, \xi)+g\left(s, x\left(s-r_{1}, \xi\right)\right)\right] d s \\
&+f\left(t, x\left(t-r_{2}, \xi\right)\right)+\sigma(t) \frac{d B^{H}}{d t}(t) \\
& x(t, 0)+g\left(t, x\left(t-r_{1}, 0\right)\right)=0 \quad \text { for } \quad t \geq 0 \text { for } t \geq 0 \\
& x(t, \pi)+g\left(t, x\left(t-r_{1}, \pi\right)\right)=0 \\
& x(\theta, \xi)=x_{0}(\theta, \xi), \varphi(s, .) \in L^{2}[0, T], \quad-\tau \leq \theta \leq 0, \quad 0 \leq \xi \leq \pi
\end{aligned}\right.
$$

where $B^{H}$ denotes a fractional Brownian motion, $g, f: \mathbb{R}^{+} \times \mathbb{R} \rightarrow \mathbb{R}$, and $b: \mathbb{R}^{+} \rightarrow \mathbb{R}$ are continuous functions. Let $Y=L^{2}([0, \pi])$ and $e_{n}:=\sqrt{\frac{2}{\pi}} \sin (n x), \quad(n=1,2,3, \cdots)$. 
Then $\left(e_{n}\right)_{n \in \mathbb{N}}$ is a complete orthonormal basis in $Y$. Let $X=L^{2}([0, \pi])$ and $A=\frac{\partial^{2}}{\partial z^{2}}$, with domain $D(A)=H^{2}([0, \pi]) \cap H_{0}^{1}([0, \pi])$. Then, it is well known that $A z=-\sum_{n=1}^{\infty} n^{2}\left\langle z, e_{n}\right\rangle e_{n}$ for any $z \in X$, and $A$ is the infinitesimal generator of a strongly continuous semigroup of bounded linear operators $\{T(t)\}_{t \geq 0}$ on $X$, which is given by $T(t) \phi=\sum_{n=1}^{\infty} e^{-n^{2} t}\left\langle\phi, e_{n}\right\rangle e_{n}, \phi \in D(A)$. In order to define the operator $Q: Y \rightarrow Y$, we choose a sequence $\left\{\sigma_{n}\right\}_{n \geq 1} \subset \mathbb{R}^{+}$and set $Q e_{n}=\sigma_{n} e_{n}$, and assume that $\operatorname{tr}(Q)=\sum_{n=1}^{\infty} \sqrt{\sigma_{n}}<\infty$. Define the process $B^{H}(s)$ by

$$
B^{H}=\sum_{n=1}^{\infty} \sqrt{\lambda_{n}} \gamma_{n}(t) e_{n}
$$

where $H \in\left(\frac{1}{2}, 1\right)$ and $\left\{\gamma_{n}^{H}\right\}_{n \in \mathbb{N}}$ is a sequence of two-sided one-dimensional fractional Brownian motions mutually independent. We suppose that

(i) For $t \geq 0, f(t, 0)=g(t, 0)=0$.

(ii) There exists a positive constant $l_{1}$, such that

$$
\left|f\left(t, \zeta_{1}\right)-f\left(t, \zeta_{2}\right)\right| \leq l_{1}\left|\zeta_{1}-\zeta_{2}\right|
$$

for $t \geq 0$ and $\zeta_{1}, \zeta_{2} \in \mathbb{R}$;

(iii) There exists a positive constant $l_{2}$, such that

$$
|f(t, \zeta)| \leq l_{2}\left(1+|\zeta|^{2}\right)
$$

for $t \geq 0$ and $\zeta \in \mathbb{R}$;

(iv) There exists a positive constant $l_{3}, 0<\pi l_{3}^{2}<1$, such that

$$
\left|g\left(t, \zeta_{1}\right)-g\left(t, \zeta_{2}\right)\right| \leq l_{3}\left|\zeta_{1}-\zeta_{2}\right|
$$

for $t \geq 0$ and $\zeta_{1}, \zeta_{2} \in \mathbb{R}$;

(v) There exists a positive constant $l_{4}$, such that

$$
|g(t, \zeta)| \leq l_{4}\left(1+|\zeta|^{2}\right)
$$

for $t \geq 0$ and $\zeta \in \mathbb{R}$;

(vi) The function $\sigma:\left[0,+\infty\left[\rightarrow \mathcal{L}_{2}^{0}\left(L^{2}([0, \pi]), L^{2}([0, \pi])\right)\right.\right.$ satisfies

$$
\int_{0}^{T}\|\sigma(s)\|_{\mathcal{L}_{2}^{0}}^{2} d s<\infty, \quad \forall T>0 .
$$

For $t \geq 0$ and $\phi \in X$, define the operators $F, G: \mathbb{R}^{+} \times X \rightarrow X$ for $\xi \in[0, \pi]$ by

$$
\begin{gathered}
G(t, \phi)(\xi)=g\left(t, \phi\left(-r_{1}\right)(\xi)\right), \quad \text { for } \quad \xi \in[0, \pi] \quad \text { and } \quad \phi \in X, \\
F(t, \phi)(\xi)=f\left(t, \phi\left(-r_{2}\right)(\xi)\right), \quad \text { for } \quad \xi \in[0, \pi] \quad \text { and } \quad \phi \in X .
\end{gathered}
$$


If we put

$$
\left\{\begin{array}{l}
u(t)(\xi)=x(t, \xi) \text { for } t \geq 0 \text { and } \xi \in[0, \pi] \\
\varphi(\theta)(\xi)=x_{0}(\theta, \xi) \text { for } \theta \in[-r, 0] \text { and } \xi \in[0, \pi]
\end{array}\right.
$$

then Eq. (4.1) takes the following abstract form

$$
\left\{\begin{aligned}
d[u(t) & \left.+G\left(t, u\left(t-r_{1}\right)\right)\right]=A\left[u(t)+G\left(t, u\left(t-r_{1}\right)\right)\right] d t \\
& +\left[\int_{0}^{t} B(t-s)\left[u(s)+G\left(s, u\left(s-r_{1}\right)\right)\right] d s+F\left(t, u\left(t-r_{2}\right)\right)\right] d t \\
& +\sigma(t) d B^{H}(t) \text { for } t \geq 0, \\
u(t)=\varphi, \quad t \in[-r, 0] &
\end{aligned}\right.
$$

Moreover, if $b$ is a bounded and $C^{1}$ function such that $b^{\prime}$ is bounded and uniformly continuous, then (H1) and (H2) are satisfied and hence, by Theorem 2.2, Eq. (4.1) has a resolvent operator $(R(t))_{t \geq 0}$ on $X$. As a consequence of the continuity of $f$ and $g$ and assumption (i) it follows that $F$ and $G$ are continuous on $\mathbb{R}^{+} \times X$ with values in $X$. By assumption (ii), one can see that

$$
\left\|F\left(t, \phi_{1}\right)-F\left(t, \phi_{2}\right)\right\|_{X} \leq \pi L_{1}\left\|\phi_{1}-\phi_{2}\right\|_{X},
$$

demonstrating that $F(t, u)$ satisfies a Lipschitz condition. Similarly, it can also be verified the same property for $G(t, u)$.

Furthermore, by assumption (iii) it follows that $\left\|F\left(t, \phi_{1}\right)\right\|_{X} \leq l_{2} \pi\left(1+\left\|\phi_{1}\right\|_{X}^{2}\right), \quad t \geq 0$. The remaining conditions can be verified similarly. Thus, all the assumptions of Theorem 3.3 are fulfilled. Therefore, the existence of a unique mild solution of Eq. (4.1) follows.

Acknowledgements. We would like to thank the referee for helpful suggestions which allowed us to improve the presentation of this paper. This research has been partially supported by FEDER and Ministerio de Economía y Competitividad (Spain) under grant MTM2011-22411

\section{References}

[1] B. Boufoussi, S. Hajji, Neutral stochastic functional differential equations driven by a fractional Brownian motion in a Hilbert space, Statistics and Probability Letters, 82 (2012), 1549-1558.

[2] J. Bao, Z. Hou, Existence of mild solutions to stochastic neutral partial functional differential equations with non-Lipschitz coefficients, Computers and Mathematics with Applications 59 (2010), 207-214.

[3] S. Tindel, C. Tudor, F. Viens, Stochastic evolution equations with fractional brownian motion, Probab. Theory Related Fields 127 (2) (2003), 186-204

[4] D. Nualart, The Malliavin Calculus and Related Topics, second ed. Springer-Verlag,2006

[5] R. Grimmer, Resolvent operators for integral equations in a Banach space, Trans. Amer. Math. Soc., 273 (1982), 333-349.

[6] R. Grimmer, A. J. Pritchard, Analytic resolvent operators for integral equations, J. Diferential Equations, 50 (1983), 234-259. 
[7] T.E. Govindan, Almost sure exponential stability for stochastic neutral partial functional differential equations, Stochastics 77 (2005) 139-154.

[8] T. Taniguchi, Successive approximations of solutions of stochastic differential equations, J. Differential Equations 96 (1992), 152-169.

[9] T. Caraballo, J. Real, T. Taniguchi, The exponential stability of neutral stochastic delay partial differential equations, Discrete Contin. Dyn. Syst. 18(2007), 295-313.

[10] T. Caraballo, M.J. Garrido-Atienza, T. Taniguchi, The existence and exponential behavior of solutions to stochastic delay evolution equations with a fractional Brownian motion, Nonlinear Anal 74 (2011), 3671-3684.

[11] E. Alos, O. Mazet, D. Nualart, Stochastic calculus with respect to Gaussian processes, Ann. Probab. 29 (1999), 766-801.

[12] Y. Mishura, Stochastic Calculus for Fractional Brownian Motion and Related Topics, in: Lecture Notes in Mathematics, 1929, 2008.

[13] J. Turo, Successive approximations of solutions to stochastic functional differential equations, Period. Math.Hungar. 30 (1995) 87-96.

[14] D. Nualart, The Malliavin Calculus and Related Topics, second edition, Springer-Verlag, Berlin, 2006.

[15] G. Cao, K. He, X. Zhang, Successive approximations of infinite dimensional SDES with jump, Stoch. Syst. 5 (2005), 609-619.

[16] F. Jiang, Y. Shen, A note on the existence and uniqueness of mild solutions to neutral stochastic partial functional differential equations with non-Lipschitz coefficients, Computers and Mathematics with Applications 61 (2011), 1590-1594.

[17] K. Liu, Stability of Infinite Dimensional Stochastic Differential Equations with Applications, Chapman and Hall, CRC, London, 2006.

[18] G. Da Prato, J. Zabczyk, Stochastic equations in infinite dimensions, Cambridge University Press, Cambridge, 1992. 